\title{
Isolation, Molecular Identification and Antibiogram of Streptococcus dysgalactiae Isolates Recovered from Pigs
}

\author{
Renu Chauhan $^{1 *}$, Lahari Laddika ${ }^{1}$, M. Dinesh ${ }^{2}$, Bhutediya Jitendrakumar Maganbhai ${ }^{1}$, \\ Shumaila Malik ${ }^{1}$, M. Sahoo ${ }^{2}$, Salauddin Qureshi ${ }^{1}$ and A. K. Tiwari ${ }^{1}$ \\ ${ }^{1}$ Division of Biological Standardisation, ICAR-Indian Veterinary Research Institute, \\ Izatnagar, Bareilly, India \\ ${ }^{2}$ Division of Pathology, ICAR- Indian Veterinary Research Institute, Izatnagar, Bareilly, India \\ *Corresponding author
}

\section{A B S T R A C T}

\begin{tabular}{|l|}
\hline Ke y w or d s \\
Molecular \\
identification \\
Antibiogram, \\
$\begin{array}{l}\text { Streptococcus } \\
\text { dysgalactiae, Pigs }\end{array}$ \\
\hline Article Info \\
\hline $\begin{array}{l}\text { Accepted: } \\
\text { 22 November } 2020 \\
\text { Available Online: } \\
\text { 10 December } 2020\end{array}$ \\
\hline
\end{tabular}

Streptococcus dysgalactiae is a pyogenic species pathogenic both for animals and humans. It is an emerging pathogen classified as $\beta$ haemolytic streptococci belonging to Lancefield group $\mathrm{C}$ and $\mathrm{G}$. Present study was undertaken to isolate, identify and analyse the antibiogram of $S$. dysgalactiae isolates recovered from pigs. A total of 53 samples were collected from slaughtered pigs, out of which 35 isolates confirmed as Gram positive streptococci microscopically. Biochemically 31 isolates were identified as Streptococcus dysgalactiae subspequisimilis and 4 as group L Streptococci by using API STREP 20multitest kit identification system. PCR targeting 23S rDNA gene sequence which confirmed 31 isolates as Streptococcus dysgalactiae. Antibiogram of these isolates was done using 13 antibiotic discs for testing antimicrobial sensitivity using standard disc diffusion method. All the isolates were highly sensitive to $\beta$ lactams followed by fluoroquinolones, tetracyclines, least susceptible to sulphonamides, aminoglycosides and were complete resistant to neomycin. Hence the study highlights the presence of emerging opportunistic pathogen $S$. dysgalactiae in pigs and its decreasing susceptibility towards aminoglycosides. This study is significant as microorganism Streptococcus dysgalactiae subsp equisimilis is an emerging pathogen in humans as well.

\section{Introduction}

Streptococcus dysgalactiae is a gram positive, beta haemolytic cocci belonging to family Streptococcaceae. Streptococcus dysgalactiae infects humans and as well as animals. Streptococcus dysgalactiae is currently divided into the subspecies Streptococcus dysgalactiae subspecies equisimilis and Streptococcus dysgalactiae subspecies dysgalactiae; the former mostly associated with human disease, and the latter almost exclusively encountered in veterinary medicine (VIEIRA et al., 1998).

The clinical manifestations in human disease range from superficial skin-infections and tonsillitis, to severe necrotising fasciitis and bacteraemia (Hughes et al., 2009; Sri et al., 2018). 
Animal species are susceptible to infection by $S$. dysgalactiae, but bovine mastitis and infectious arthritis in lambs (joint ill) have been most frequently reported (Whist et al., 2007; Rutherford et al., 2017). In veterinary medicine, retrospective examination of bibliographic data about Streptococcus dysgalactiae subspecies equisimilis infection is complicated by the frequent nomenclature changes due to the animal origin of the strains (Preziuso et al., 2010). S. equisimilis has been isolated infrequently from placentas from aborted, stillborn, and premature foals (Hong et al., 1993).

Endocarditis and arthritis due to S. equisimilis infection have been recently described in swine (Kawata et al., 2003), while the data published before refer to isolation of $S$. dysgalactiae without subspecies distinction (Hong et al., 1993). Furthermore, considering the lack of information, it is also difficult to evaluate if the animals play a role in the maintenance and transmission of this potential zoonotic agent.

A PCR identification system targeting 23S rDNA sequences for the identification of eight streptococcal species relevant to animal infections (S.agalactiae, S. bovis, S. canis, S. dysgalactiae, S. equi, S. porcinus, S. suis and $S$. uberis) was used in the present study(Kawata et al., 2004).

The aim of present study was identification of isolates recovered from pigs by biochemical and molecular method of identification followed by antibiogram.

\section{Materials and Methods}

\section{Sample collection}

Total 53 samples were collected from pigs which were slaughtered at slaughter house of Bareilly. Heart blood was collected in sterile vaccutainer. Lymph nodes and joint fluid samples were also taken.

\section{Sample processing}

Samples were inoculated in BHI broth (Difco) and kept for incubation at $37^{\circ} \mathrm{C}$ for $4-6$ hours. Loopful of culture was then streaked on $5 \%$ sheep blood agar. After overnight incubation under micro-aerophilic condition cultural characteristics were observed. Isolates which showed $\beta$-haemolytic colonies (showing complete haemolysis) were picked up and further subcultured on blood agar.

\section{Culture identification}

Cultural characteristics were observed followed by microscopic morphology was observed by Gram's staining method.

\section{Biochemical characterization}

Bacterial isolates were subjected to catalase test and oxidase test. All catalase negative isolates were further screened by API 20 STREP (bioMerieux) multitest kit identification system as per manufacturer's instruction.

\section{Molecular identification}

DNA isolation was done by snap chilled method. Quality and purity of DNA was determined by taking absorbance at 260 and $280 \mathrm{~nm}$ in UV spectrophotometer. A PCR identification system targeting 23S rDNA sequences (Kawata et al., 2004) was used to screen the recovered isolates.

\section{Antibiotic sensitivity testing}

Antibiotic sensitivity assay of characterized isolates was performed as per CLSI guidelines with slight modifications.Colony suspension, equivalent to a 0.5 McFarland 
standard, prepared using colonies from an overnight (18 to $20 \mathrm{hr}$ ) sheep blood agar plate at $37{ }^{\circ} \mathrm{C}$. Disk diffusion method was used on MHA with 5\% sheep blood.

Zone of inhibition was measured after 18-20 hrs of incubation at $37{ }^{\circ} \mathrm{C}$ under microaerophilic condition.

\section{Results and Discussion}

\section{Culture identification}

Out of 53 samples 35 pure isolates were obtained which showed clear $\beta$ haemolysis on blood agar. Colonies were round, whitish to grey, 1-2 $\mathrm{mm}$ in size, raised with entire edge. Microscopically Gram positive cocci present in pairs and chain were seen (Fig. 1 and 2).

\section{Biochemical characterization}

All the isolates were oxidase and catalase negative. 31 Isolates were biochemically characterized as Streptococcus dysgalactiae subspecie sequisimilis and 4isolates as $\mathrm{L}$ group streptococci by API 20 STREP mutitiest kit identification system (BioMerieux). Specific number obtained for each isolate which used to identify isolate by apiweb software.

Fig.1 API kit result after 24 hours of incubation, colour changes in reagents observed for individual cupule

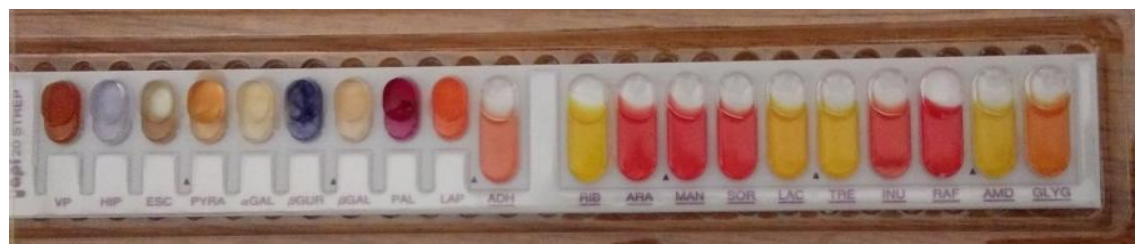

Fig.2 PCR product of 23S rDNA gene of field isolates confirmed as $S$. dysgalactiae

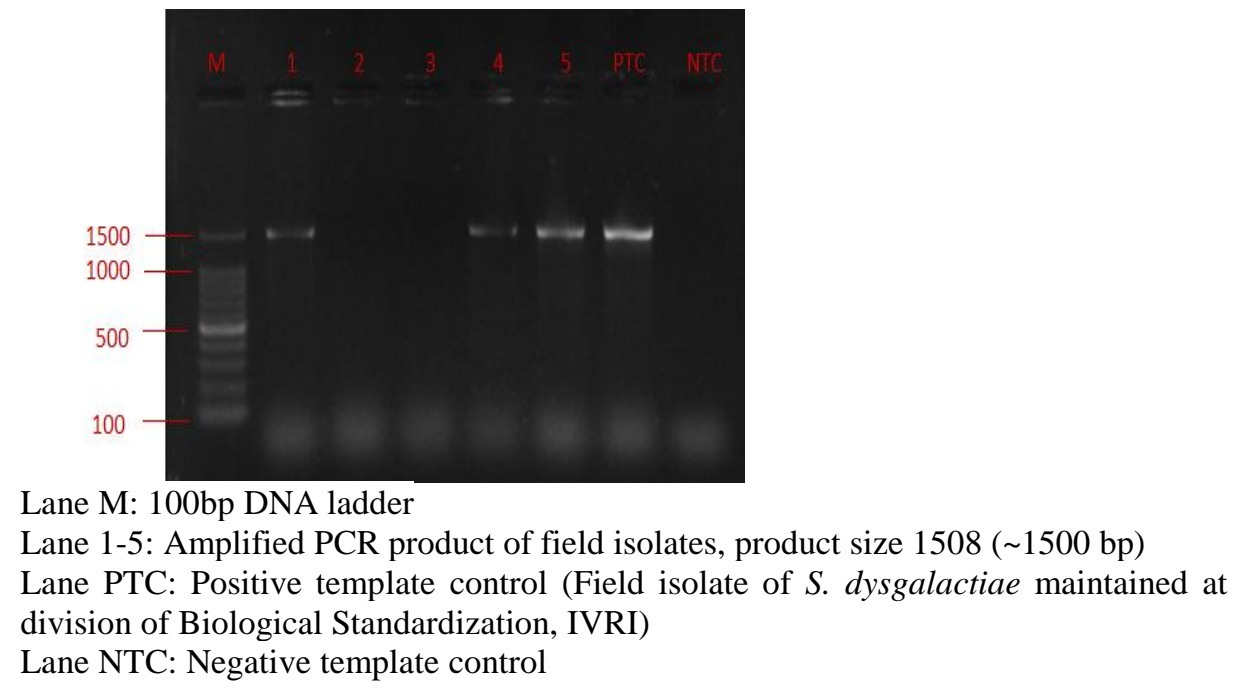

\section{Molecular identification}

Out of 35 isolates 31 isolates were identified as Streptococcus dysgalactiae after screening through reaction $\mathrm{A}$ and $\mathrm{B}$ as described by Kwata et al., (2004). A product size of 1508 bp was amplified for $S$. dysgalactiae. DNA of the rest of the 4 isolates were not amplified in 
either of the reaction, therefore could not be identified.

\section{Antibiotic sensitivity}

All isolates were highly sensitive to $\beta$ lactam antibiotics, among which wide zone of inhibition was seen for ampicillin and cloxacillin. Resistance was shown by 18 isolates for streptomycin and 24 isolates were resistant to amikacin $80 \%$ isolates showed resistance for sulphadiazine and all the isolates were resistant for neomycin.

S. dysgalactiae can infect several animal species whereas, Streptococcus dysgalactiae subspecies equisimilis can infect humans as well as animals. Currently, bacterial culture and biochemical identification with commercial kits are widely used for $S$. dysgalactiae diagnosis. Isolated $\beta$-haemolytic colonies were obtained on 5\%sheep blood agar which was a parameter of presumptive identification (Baeck et al., 2006; Ciszewsk iet al., 2016). 31 isolates were confirmed as Streptococcus dysgalactiae subspecies equisimilis by using API 20 STREP mutitiest kit identification system (BioMerieux), This API multitest kit system has been used earlier by various workers for the biochemical characterization of $S$. dysgalactiae (Poutrel et al., 1984; Baeck et al., 2006). Nevertheless, overgrowing of other beta-haemolytic streptococci or mistakes in biochemical profile interpretation may be encountered during bacterial examination. PCR is widely used in many fields of microbiology.

In PCR S.dysgalactiae was identified successfully as described by Kwata et al., (2004) A product size of $1508 \mathrm{bp}$ was obtained.

The isolates of $S$. dysgalactiae were susceptible to $\beta$-lactams and this finding was similar to the previous studies (Tsi et al.,
2014; Ciszewski et al., 2016; Lu et al., 2016). However resistance was seen for streptomycine, amikacin and sulphadiazine in increasing order while complete resistance was seen for neomycine.

In conclusion the isolates characterized as $S$. dysgalactiae by PCR system. Same isolates characterized as $S$. dysgalactiae subsp. equisimilis by API multitest kit identification system. Antibiogram revealed decreasing sensitivity for aminoglycosides. Resistant to commonly used antibiotic azithromycin in upper respiratory tract infection was observed.

\section{References}

Baeck, G.W., Kim, J.H., Gomez, D.K. and Park, S.C., 2006. Isolation and characterization of Streptococcus sp. from diseased flounder (Paralichthys olivaceus) in Jeju Island. Journal of veterinary science, 7(1), pp.53-58.

Ciszewski, M., Zegarski, K. and Szewczyk, E.M., 2016. Streptococcus dysgalactiae subsp. equisimilis isolated from infections in dogs and humans: are current subspecies identification criteria accurate? Current microbiology, 73(5), pp.684-688.

Hong, C.B., Donahue, J.M., Giles Jr, R.C., Petrites-Murphy, M.B., Poonacha, K.B., Roberts, A.W., Smith, B.J., Tramontin, R.R., Tuttle, P.A. and Swerczek, T.W., 1993. Etiology and pathology of equine placentitis. Journal of Veterinary Diagnostic Investigation, 5(1), pp.5663.

Hughes, J.M., Wilson, M.E., Brandt, C.M. and Spellerberg, B., 2009. Human infections due to Streptococcus dysgalactiae subspecies equisimilis. Clinical Infectious Diseases, 49(5), pp.766-772.

Kawata, K., Anzai, T., Senna, K., Kikuchi, 
N., Ezawa, A. and Takahashi, T., 2004. Simple and rapid PCR method for identification of streptococcal species relevant to animal infections based on 23S rDNA sequence. FEMS microbiology letters, 237(1), pp.57-64.

Kawata, K., Minakami, T., Mori, Y., Katsumi, M., Kataoka, Y., Ezawa, A., Kikuchi, N. and Takahashi, T., 2003. rDNA sequence analyses of Streptococcus dysgalactiae subsp. equisimilis isolates from pigs. International journal of systematic and evolutionary microbiology, 53(6), pp.1941-1946.

Lu, B., Fang, Y., Huang, L., Diao, B., Du, X., Kan, B., Cui, Y., Zhu, F., Li, D. and Wang, D., 2016. Molecular characterization and antibiotic resistance of clinical Streptococcus dysgalactiae subsp. equisimilis in Beijing, China. Infection, Genetics and Evolution, 40, pp.119-125.

Poutrel, B.E.R.N.A.R.D. and Ryniewicz, H.Z., 1984. Evaluation of the API 20 Strep system for species identification of streptococci isolated from bovine mastitis. Journal of Clinical Microbiology, 19(2), pp.213-214.

Preziuso, S., Laus, F., Tejeda, A.R., Valente, C. and Cuteri, V., 2010. Detection of Streptococcus dysgalactiae subsp. equisimilis in equine nasopharyngeal swabs by PCR. Journal of veterinary science, 11(1), pp.67-72.
Rutherford, S.J., Rycroft, A.N. and Ridler, A.L., 2014. Sources of Streptococcus dysgalactiae in English and Welsh sheep flocks affected by infectious arthritis (joint ill). Veterinary Record.

Sri, C.S., Kamleshun, R., Barnett, B.J., Mejias, S.G. and Petras, L., 2018. A Rare Case of Streptococcus dysgalactiae Subsp. Dysgalactiae Human Zoonotic Infection. Cureus, 10(7).

Tsai, C.T., Chi, C.Y., Ho, C.M., Lin, P.C., Chou, C.H., Wang, J.H., Wang, J.H., Lin, H.C., Tien, N., Lin, K.H. and Ho, M.W., 2014. Correlation of virulence genes to clinical manifestations and outcome in patients with Streptococcus dysgalactiae subspecies equisimilis bacteremia. Journal of Microbiology, Immunology and Infection, 47(6), pp.462-468.

Vieira, V.V., Teixeira, L.M., Zahner, V., Momen, H., Facklam, R.R., Steigerwalt, A.G., Brenner, D.J. and Castro, A.C., 1998. Genetic relationships among the different phenotypes of Streptococcus dysgalactiae strains. International Journal of Systematic and Evolutionary Microbiology, 48(4), pp.1231-1243.

Whist, A.C., Østerås, O. and Sølverød, L., 2007. Streptococcus dysgalactiae isolates at calving and lactation performance within the same lactation. Journal of dairy science, 90(2), pp.766778.

\section{How to cite this article:}

Renu Chauhan, Lahari Laddika, M. Dinesh, Bhutediya Jitendrakumar Maganbhai, Shumaila Malik, M. Sahoo, Salauddin Qureshi and Tiwari, A. K. 2020. Isolation, Molecular Identification and Antibiogram of Streptococcus dysgalactiae Isolates Recovered from Pigs. Int.J.Curr.Microbiol.App.Sci. 9(12): 3026-3030. doi: https://doi.org/10.20546/ijcmas.2020.912.358 